\title{
Tratamiento de desechos del cianuro por biorremediación
}

Alma Deloya Martínez'

Fecha de recepción: 4 de abril del 2011 Fecha de aceptación: 6 de setiembre del 2011

Deloya, A. Tratamiento de desechos del cianuro por biorremediación. Tecnología en Marcha. Vol. 25, № 2. Abril-Junio 20 I2. Pág 6I-72. 


\section{Resumen}

Se presentan los resultados de una investigación en la que se desarrolló un consorcio autóctono de microorganismos degradadores del cianuro para aplicarlo en el tratamiento biológico de los desechos peligrosos del cianuro.

Los microorganismos autóctonos obtenidos se liofilizaron en diferentes medios protectores, como la gelatina y caldo lactosado a diferentes temperaturas $(-35,-45,-55$ y -65$)$.

Para el tratamiento preliminar de los desechos del cianuro se aplicó un método de pretratamiento en lechada: para la lixiviación preliminar del desecho, con periodos de 3-5 días y un tratamiento posterior, por lagunas aireadas, aplicando el consorcio de microorganismos liofilizados.

Se obtuvieron ocho diferentes liofilizados bajo diferentes condiciones de temperatura y con dos medios protectores de liofilización que presentaron excelentes recuperaciones a los seis meses de la liofilización.

El consorcio de microorganismos liofilizados presentó, viabilidad del 70 al 80\%, con porcentajes de remoción del cianuro mayores al $95 \%$ y puede conservarse activo por tiempos prolongados (por años).

Los microorganismos liofilizados pueden aplicarse en la biodegradación de los desechos del cianuro procedentes de las minas de oro o cualquier otro desecho de cianuro como los baños de electrodeposición de metales, además de aquellos procedentes de la industria manufacturera de joyas.

\section{Palabras clave}

Microorganismos autóctonos, medio protector, liofilización, lixiviación, consorcio de microorganismos, biorremediación.

\section{Abstract}

In the following research was developed an autochthonous consortium of degrader microorganisms of the cyanide for the application in the biological treatment of the dangerous wastes of cyanide.

The autochthonous microorganisms obtained were lyophilized in different protective environments such as gelatin and lactose broth at different temperatures $(-35,-45,-55$ y -65$)$.

For the preliminary treatment of the cyanide wastes it was applied a method for the preliminary leaching of the waste, with periods between 3 and 5 days, and a posterior treatment, by aerated lagoons, applying the consortium of lyophilized microorganisms.

There were obtained eight different lyophilized in different temperature conditions and with two lyophilization protective media that presented excellent recovery at six months of lyophilization.

The consortium of lyophilized microorganisms presented 70 to 80 percent of viability, with cyanide removal percentages higher than 95 percent and it can be conserved active for a prolonged time (for years).

The lyophilized microorganisms can be applied in the biodegradation of the cyanide wastes from the gold mines or any other cyanide waste such as metal plating baths as well as from jewelry manufacturing.

\section{Key words}

Autochthonous microorganisms, protective media, lyophilization (Freeze drying), leaching, consortium of microorganisms, biodegradation. 


\section{Introducción}

Actualmente, los desechos del cianuro son tratados por métodos químicos muy eficientes y con buenos resultados. Sin embargo, la búsqueda de tecnologías correctivas biológicas para degradar el cianuro, que no generen productos tóxicos y que sean menos impactantes para el ambiente, ha impulsado las investigaciones para sustituir los métodos convencionales como son la adsorción, la conversión química y el tratamiento electrolítico. Estos son costosos y corrosivos, además de que generan desechos que a veces son más contaminantes que los iniciales.

El planteamiento de este proyecto surge con el objetivo de adaptar tecnología que sea más amigable con el ambiente, como la biorremediación.

Una de las ventajas del tratamiento de los desechos tóxicos por biorremediación es que es más económico por cuanto no se requiere de reactivos químicos. Estos últimos implican un manejo adecuado y, en la mayoría de los casos, costos elevados. Además, la biorremediación es un método sencillo, no necesita de personal experto para el manejo y operación del sistema de tratamiento.

La biorremediación causa impacto menos negativo en el medio ambiente. Está basada en la acción de microorganismos autóctonos y modificaciones ambientales de gran sencillez, como la aplicación de nutrientes y la aireación.

Por ser el cianuro biodegradable, sus desechos pueden ser tratados por biorremediación, ya que la colonización y crecimiento de los microorganismos no será inhibida por altas concentraciones, si el desecho es acondicionado con un pre tratamiento como el de lechada (lixiviación en agua con agitación).

Durante el tratamiento por biorremediación, el cianuro se transforma en otras sustancias químicas más estables y menos tóxicas mediante procesos físicos, químicos y biológicos naturales.

Los factores que intervienen en el proceso de biorremediación son variados y entre los más importantes para la biorremediación del cianuro en medio acuoso son el pH, el oxígeno y por último la concentración del cianuro.

En el proceso de investigación se desarrolló un consorcio de microorganismos autóctonos para degradar el cianuro y aplicarlo en el tratamiento biológico de los desechos del cianuro (biorremediación). El tratamiento preliminar consistió en la lixiviación del desecho y un posterior tratamiento aerobio por Lagunas aireadas a $\mathrm{pH}$ no menor a 9,4.

\section{Planteamiento del problema}

Verter los residuos líquidos industriales del cianuro en la naturaleza sin ningún tratamiento sobrepasa la capacidad de auto-recuperación de los cuerpos receptores, o del suelo sobre el cual se disponen, ya que alteran sus características y provocan una eminente contaminación y gran impacto ambiental, al destruir ecosistemas y afectar la salud de poblaciones.

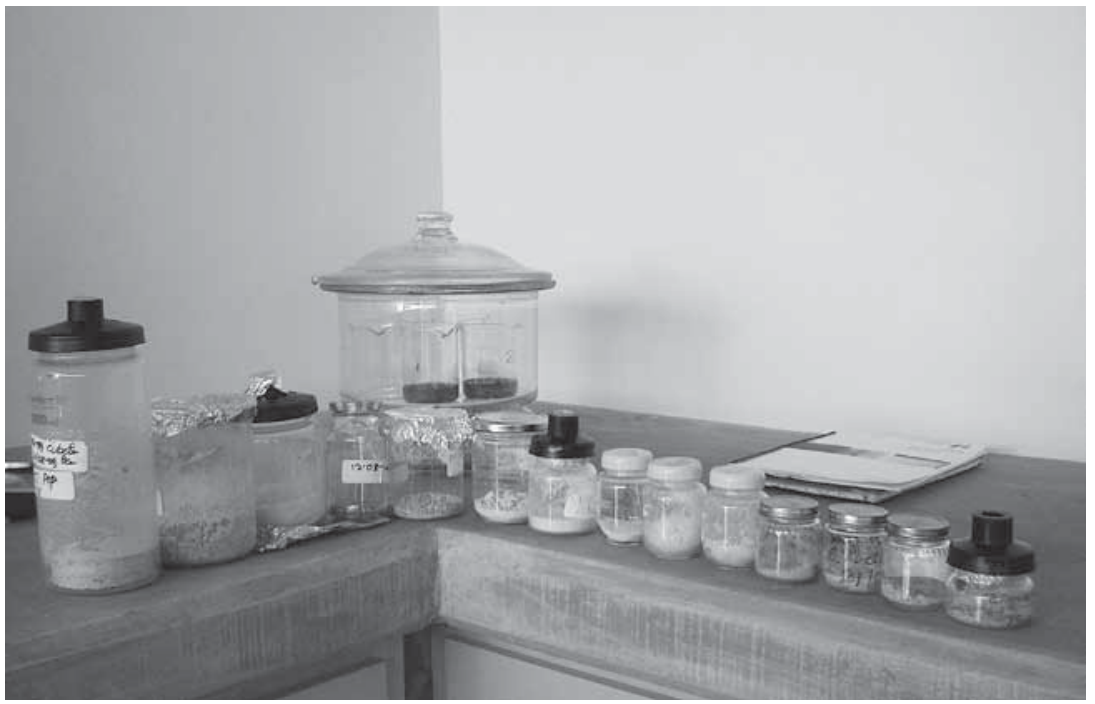

Figura I. Liofilizados del consorcio de microorganismos, a diferentes temperaturas de liofilización después de 10 meses de preservación. 
El tratamiento de los contaminantes en el agua, permite proteger el medio ambiente y la salud, así como cumplir con las especificaciones de vertido del reglamento 3360 I MINSALUD-MINAE que rige en Costa Rica.

Generalmente los tratamientos de los desechos del cianuro se han realizado por métodos químicos costosos que requieren de sustancias químicas que implican alta inversión y una adecuada dosificación y manipulación.

Este es el caso de los efluentes derivados de los procesos que utilizan grandes cantidades de cianuro. Por ejemplo el proceso de recuperación de metales preciosos de oro y plata a partir de yacimientos de rocas intrusitas de vetas de cuarzo, presentan reacciones colaterales con los minerales asociados al oro, o la plata, que son bastante complejas y tóxicas.

Además, en el Laboratorio de Ingeniería de los Materiales del Tecnológico de Costa Rica se generan desechos del proceso de nitruración de metales que pueden tratarse por medio de la biodegración por medio de la aplicación del consorcio de microorganismos degradadores del cianuro obtenido, producto de esta investigación.

\section{Objetivo general}

Desarrollar un consorcio de microorganismos adaptado para degradar concentraciones elevadas de cianuro, que se conserve por tiempos prolongados, para aplicarlos en la biorremediación de los desechos peligrosos del cianuro.

\section{Objetivos específicos}

- Aplicar el pre tratamiento por lechada para la lixiviación y desintoxicación de desechos del cianuro.

- Obtener un extracto enzimático o microbiano por la técnica del sustrato selectivo, para aplicarlo en la biorremediación de los desechos del cianuro.

- Secar el extracto obtenido por liofilización, para conservarlo y disponerlo para su aplicación por periodos prolongados (por años).

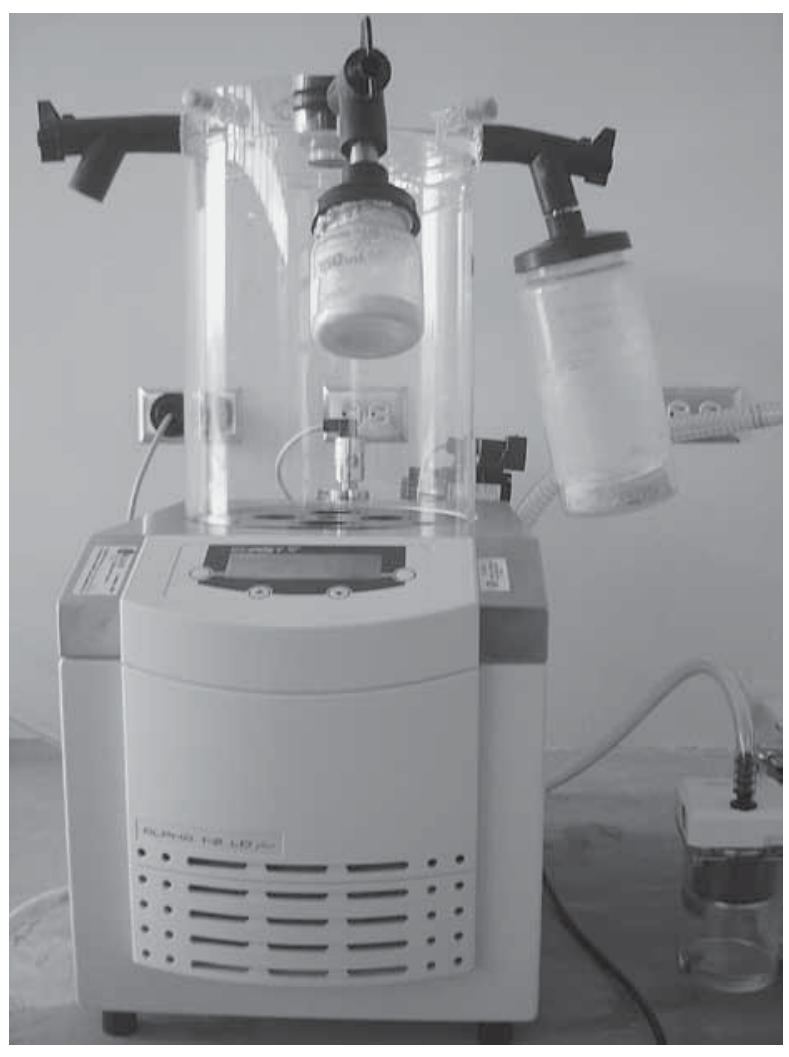

Figura 2. Para la liofilización se utilizó el Liofilizador - Freeze Dyer ALPHA 2-4 / LD.

\section{Materiales y métodos}

Se obtuvo un consorcio de microorganismos a partir de un desecho de cianuro generado durante el proceso de nitruración' en el laboratorio de ingeniería de los materiales del Tecnológico de Costa Rica, sede Cartago.

\section{Equipo principal}

El contenido de cianuro en el desecho se midió con el Espectrofotómetro portátil, LaMotte SMART Spectro.

La nitruración líquida se realiza en un baño de sales fundidas compuesto de una mezcla típica de sales de sodio y potasio. Las sales de sodio representan de un 60 a un 70\% (en peso) de la mezcla total, están compuestas por $96.5 \%$ de $\mathrm{NaCN}, 2.5 \%$ de $\mathrm{Na}_{2} \mathrm{CO}_{3}$ y $0.5 \%$ de $\mathrm{NaCNO}$. La nitruración es, uno de los tratamientos térmicos para mejorar dureza y corrosión superficial de metales, más baratos y que menos equipamiento requiere. 
El oxígeno disuelto se determinó con un medidor con electrodo de membrana, marca Fischer Scientific, modelo APG4, portátil.

El control del pH durante el tratamiento del desecho de cianuro se midió con $\mathrm{pH}$-metro de mesa DnverUB- I0, instrumental.

Las observaciones al microcopio se realizaron con el Microscopio Trinocular, Marca MOTIC, modelo BA300.

Para la separación del extracto de microorganismo a liofilizar se usó la Centrífuga Damon y EC Division y EC- HN-FIl, modelo dcs- I 6rv-r.

Los medios bacteriológicos se esterilizaron en auto clave Biocientífica-C-75। I.

La determinación de la humedad residual del liofilizado, se realizó en estufa Marca Thermo Electron Corportion, Precision.

\section{Metodología}

El desecho en estudio se caracterizó en cuanto al contenido de cianuro, usando el método de cianuro 36-SC del espectrofotómetro portátil, LaMotte SMART Spectro.

Para la obtención del consorcio degradador del cianuro, primero se procedió a la lixiviación del desecho en agua a temperatura ambiente, con micronutrientes de calcio, hierro y magnesio, con agitación por tres días y controlando que el pH siempre fuera superior o igual a 10,4 para obtener un medio alcalino. Además se controló que el oxígeno disuelto se mantuviera por lo menos en $2 \mathrm{mg} / \mathrm{L}$, para asegurar las condiciones aerobias ( I) (2).

Posteriormente, como segundo paso para la obtención del consorcio de los microorganismos degradadores del cianuro, en los experimentos montados y previamente agitados por tres días, se prolongó la agitación por 63-72 días, bajo las condiciones mencionadas y se alimentó día por medio durante todo el periodo del ensayo, con I,4 $\mathrm{mg} / \mathrm{L}$ del desecho de cianuro, como única fuente de carbono y nitrógeno para las funciones de las bacterias. (3)

Todos los ensayos de lixiviación y biodegradación se montaron en réplicas por triplicado, en recipientes de 25 litros, con un volumen del desecho por tratar de 20 litros.

Los ensayos de control, durante el proceso de obtención del consorcio de microorganismos fueron:
$\mathrm{pH}$, oxígeno disuelto, temperatura y exámenes microscópicos. Todos los análisis se realizaron de cuerdo con los Métodos Estandarizados para el análisis de aguas y aguas residuales (4).

Para la selección y adaptación, los consorcios de microorganismos se separaron por centrifugación, a 5000 rpm, durante 10 minutos, se lavaron con agua destilada dos veces y se incubaron por 48 horas a temperatura ambiente, en caldo lactosado al 20\%. Se añadieron micronutrientes y se aumentó gradualmente la concentración de cianuro desde I 400 hasta 2500 mg/L. Los ensayos se realizaron bajo condiciones aerobias, manteniendo el oxígeno disuelto por lo menos en $2 \mathrm{mg} / \mathrm{L}$ y el $\mathrm{pH}$ entre 9,4 a 10,4 unidades de $\mathrm{pH}(\mathrm{I})$.

Este proceso de selección y adaptación de los microorganismos se llevó a cabo hasta la tercera generación de microorganismos, reinoculando los microorganismos separados por centrifugación a 5000 rpm en caldo lactosado, bajo las mismas condiciones anteriores de nutriente, oxígeno y $\mathrm{pH}$.

Se controló en todos los casos el crecimiento de los microorganismos con observaciones en el microscopio, día por medio. Las nuevas cepas obtenidas se volvieron a separar por centrifugación y se congelaron para su posterior liofilización.

Los consorcios de microorganismos, separado por centrifugación se resuspendieron en diferentes medios protectores de liofilización como son la gelatina al $10 \%$ y $5 \%$ y la lactosa al 10\%, y 5\% (5).

Después de la resuspensión, la congelación de los microorganismos, se realizó el día anterior a la liofilización por un periodo de 20 a 24 horas a temperaturas de -3 a 0 grados centígrados.

El proceso de liofilización se realizó a presiones de $0,250,0,070,0,021$ y 0,0054 milibares equivalentes a temperaturas de resublimación entre $-35,-45,-55$ y -65 grados centígrados respectivamente. Los tiempos de liofilización fueron de 24 a 36 horas para el proceso de secado primario. El secado secundario se realizó a 20 grados centígrados, por 10 a 8 horas (6).

A los liofilizados se les determinó la humedad tanto en el secado primario como en el secado secundario, con el propósito de controlar la humedad final del liofilizado.

Los liofilizados se conservaron en refrigeración y a temperatura ambiente en ambos casos en frascos de vidrio y tapados. 
Los microorganismos liofilizados se recuperaron, rehidratándolos a temperatura ambiente, en una suspensión de caldo lactosado al $10 \%$, con una concentración de cianuro de $2500 \mathrm{mg} / \mathrm{L}, \mathrm{pH}$ de 9,4 a 10,4 y concentración de oxígeno disuelto de no menor que $2 \mathrm{mg} / \mathrm{L}$. Para la recuperación de los microorganismos liofilizados se tomaron $10 \mathrm{ml}$ al 0, I \%, de la suspensión del cultivo liofilizado y se resuspendieron en $100 \mathrm{ml}$ de caldo lactosado al 10 \%. La suspensión del liofilizado se incubó por 48 horas a temperatura ambiente (7).

El recuento heterótrofo de microorganismos, se realizó a las 48 horas de recuperación, para calcular el porcentaje de recuperación del liofilizado. El recuento heterótrofo se realizó en el Laboratorio Ceqiatec, Departamento de Química delTecnológico de Costa Rica.

El tratamiento del desecho de cianuro, se replicó por triplicado. Como pretratamiento se lixivió el desecho con volúmenes de 20 litros de agua, agitando por 3 días a 200 rpm. Durante los tres días del pretratamiento se mantuvo el $\mathrm{pH}$ en valores iguales o superiores a 10,4 unidades de $\mathrm{pH}$. Posterior a la lixiviación se trató el desecho por aireación con difusores, con las mismas condiciones de $\mathrm{pH}$, oxígeno disuelto y nutrientes con los que se obtuvo el consorcio microbiano, prolongando el tiempo tratamiento por 12 días más (9).

Se cuantificó el cianuro, antes y después del tratamiento total de 15 días, (3 días de pretratamiento por lixiviación y 12 días de tratamiento por aireación).

\section{Logros del proyecto}

El principal logro del proyecto es la obtención de un consorcio de microorganismos adaptado para tratar concentraciones elevadas de cianuro, capaz de lograr la degradación del cianuro mediante procesos biológicos, así como una biotecnología para tratar los desechos de cianuro en concentraciones similares a las generadas en las minas de oro, en procesos de electrolíticos y en joyería.

\section{Otros logros}

Además del logro principal, se obtuvieron otros resultados importantes. La generación de una nueva tecnología biológica de descontaminación de desechos tóxicos, como los desechos del cianuro; la formación de expertos en el área de biorremediación y la integración con grupos líderes en el tema de biotecnología ambiental y biorremediación de aguas en el ámbito institucional.

Asimismo, entre los impactos generados se encuentran: lograr la vinculación del Tecnológico de Costa Rica con el sector productivo minero y con empresas tecnológicas del área ambiental. Así como también la ejecución de este proyecto implica trabajar dentro de una línea estratégica de investigación importante en los temas de contaminación ambiental y en biotecnología.

Por otro lado, entre los impactos económicosociales más importantes se pueden mencionar los siguientes: en el área social, contar con un ambiente descontaminado, por el tratamiento que realizaría la industria minera y química de sus aguas residuales. En el ámbito económico, el impacto más significativo es la obtención de una solución tecnológica para el problema del tratamiento del cianuro, económicamente viable y conveniente para la realidad nacional. Asimismo, es posible lograr acuerdos de comercialización de la tecnología a nivel nacional.

\section{Resultados obtenidos}

A continuación se resumen los principales resultados del proyecto. El estudio se realizó en el Centro de Protección Ambiental de la Escuela de Química del Tecnológico de Costa Rica, en el periodo comprendido entre el 22 febrero del 2008 al 22 de febrero del 2010.

En el cuadro I se presentan las características del desecho, en cuanto a la concentración del cianuro. Seguidamente se presenta el cuadro 2 que indica el tiempo en que aparecieron los microorganismos degradadores del cianuro.

Posteriormente, en los cuadros 3 y 4 se resume el promedio de la humedad en los liofilizados, resultado del secado primario y secundario en los medios protectores de liofilización. El recuento heterótrofo, para obtener el porcentaje de recuperación de los liófilos o viabilidad, se muestra en el cuadro 5.

Por último, en el cuadro 6 se muestra el porcentaje de degradación del cianuro por el consorcio microbiano después del pretratamiento en lechada y el tratamiento con aireación y agitación. 
Cuadro I. Concentración de cianuro en el desecho del Laboratorio de Ciencias de los Materiales, TEC, Cartago.

\begin{tabular}{|c|c|}
\hline Muestra & Concentración $(\mathrm{mg} / \mathrm{L})$ \\
\hline 1 & 1400 \\
\hline 2 & 1300 \\
\hline 3 & 1200 \\
\hline 4 & 1600 \\
\hline
\end{tabular}

Fuente: Deloya, A.

Cuadro 2. Aparición de los microorganismos durante el proceso de obtención del consorcio de Microorganismos autóctonos para degradar cianuro.

\begin{tabular}{|c|c|}
\hline Muestra & Concentración $(\mathrm{mg} / \mathrm{L})$ \\
\hline 1 & 1400 \\
\hline 2 & 1300 \\
\hline 3 & 1200 \\
\hline 4 & 1600 \\
\hline
\end{tabular}

*En todos los ensayos se añadieron nutrientes: Cloruro de calcio, Cloruro de hierro y Cloruro de magnesio. Fuente: Deloya, $\mathrm{A}$.

Cuadro 3. Humedad de los liofilizados en dos medios protectores, después del secado primario.

\begin{tabular}{|c|c|c|}
\hline \multirow{2}{*}{ Liofilizado } & \multicolumn{2}{|c|}{ Medio protector } \\
\cline { 2 - 3 } & $\begin{array}{c}\text { Caldo lactosado } \\
\text { humedad (\%) }\end{array}$ & $\begin{array}{c}\text { Gelatina } \\
\text { humedad (\%) }\end{array}$ \\
\hline 1 & 11,70 & 9,09 \\
\hline 2 & 15,80 & 9,40 \\
\hline 3 & 10,00 & 8,00 \\
\hline 4 & 12,00 & 7,40 \\
\hline 5 & 14,00 & 12,00 \\
\hline
\end{tabular}

Fuente: Deloya, A.

Cuadro 4. Humedad de los liofilizados en dos medios protectores, después del secado secundario.

\begin{tabular}{|c|c|c|}
\hline \multirow{2}{*}{ Liofilizado } & \multicolumn{2}{|c|}{ Medio protector } \\
\cline { 2 - 3 } & $\begin{array}{c}\text { Caldo lactosado } \\
\text { Humedad (\%) }\end{array}$ & $\begin{array}{c}\text { Gelatina } \\
\text { Humedad (\%) }\end{array}$ \\
\hline 1 & 3,89 & 1,42 \\
\hline 2 & 2,00 & 1,20 \\
\hline 3 & 5,26 & 1,35 \\
\hline 4 & 3,00 & 1,96 \\
\hline 5 & 4,30 & 1,38 \\
\hline
\end{tabular}

Fuente: Deloya, A. 
Cuadro 5. Recuento heterótrofo para determinar la recuperación de los liofilizados. (*)

\begin{tabular}{|c|c|c|}
\hline \multirow{2}{*}{ Muestra } & \multicolumn{2}{|c|}{ Recuento ufd $\mathbf{1 0 0 m l}$} \\
\cline { 2 - 3 } & Antes de liofilizar & Después de liofilizar \\
\hline 1 & $5,9 \times 10^{17}$ & $1,5 \times 10^{15}$ \\
\hline 2 & $1,2 \times 10^{17}$ & $3,8 \times 10^{14}$ \\
\hline 3 & $2,3 \times 10^{16}$ & $3,7 \times 10^{14}$ \\
\hline
\end{tabular}

Fuente: Deloya, A.

(*) (t) tiempo de recuperación: 48 horas

Volumen de ensayo: 20 litros

$\mathrm{pH}:$ 10,4 unidades

Cuadro 6. Porcentaje de degradación del cianuro de acuerdo con las variables seleccionadas. (*)

\begin{tabular}{|c|c|c|c|}
\hline Ensayo & $\begin{array}{c}\text { Concentración inicial } \\
\text { cianuro }(\mathrm{mg} / \mathrm{L})\end{array}$ & $\begin{array}{c}\text { Concentración final } \\
(\mathrm{mg} / \mathrm{L})\end{array}$ & $\begin{array}{c}\text { Remoción } \\
(\%)\end{array}$ \\
\hline 1 & 3600 & 2,58 & 99.9 \\
\hline 2 & 1920 & 2,21 & 99,8 \\
\hline 3 & 1440 & 1,65 & 99,8 \\
\hline
\end{tabular}

Fuente: Deloya, A.

(*) (t) tiempo de recuperación: 48 horas

Volumen de ensayo: 20 litros

$\mathrm{pH}: 10,4$ unidades

\section{Discusión de resultados}

La caracterización del desecho en los diferentes lotes de las muestras, indican concentraciones de cianuro total de 1400 mg/L, I 300 mg/L, I 200 mg/L y $1600 \mathrm{mg} / \mathrm{L}$, ver cuadro I.

El pH del desecho durante los ensayos varió desde 9,48 a I I,40 unidades de $\mathrm{pH}$. Este parámetro como característica propia del desecho del cianuro, fue una ventaja y uno de los factores más importantes durante el tratamiento por biodegradación del desecho, ya que la literatura menciona que valores alcalinos de pH mejoran la degradación del cianuro (9).

El cianuro se puede presentar en dos formas, dependiendo del $\mathrm{pH}$ del medio en el que se encuentre. Con $\mathrm{pH}$ alcalino predomina la forma soluble del cianuro, mientras que con un $\mathrm{pH}$ neutro y ácido en el medio, predomina la forma gaseosa, el ácido cianhídrico (10).

Por lo anterior, durante el tratamiento por biodegradación del cianuro, se trabajó manteniendo el $\mathrm{pH}$ con valores entre 9,16 a 10,4. Esto es de vital importancia para que el cianuro permanezca disuelto y no pase al medio ambiente como HCN gaseoso, porque este gas actúa en pocos segundos en el sistema respiratorio, inhibiendo enzimas respiratorias como la citocromo oxidasa (I I).

Durante el proceso de tratamiento en lechada se extrajo el cianuro sólido mediante la agitación usando como disolvente el agua. El cianuro entra en contacto con el agua al ser agitado y se difunde a la fase líquida, con lo que se logra que el cianuro sólido pase a la fase liquida. Este proceso preliminar en el tratamiento por biodegradación del cianuro también se conoce como lixiviación del cianuro (2).

La agitación para mantener el contacto entre el desecho y el agua mostró ser de vital importancia para realizar la biodegradación del desecho porque permite la lixiviación del cianuro por el agua (12).

El desarrollo y obtención de los microorganismos nativos degradadores del cianuro se logró en periodos muy prolongados. El desarrollo de los primeros microorganismos se encontró a los 72 días, 
en el primer ensayo; en la segunda réplica, a los 66 días; en la tercera réplica, a los 63 días y en la última, a los 60 días (ver cuadro 2). Este tiempo prolongado para la aparición de los microorganismos autóctonos degradadores del cianuro, en los diferentes reactores de ensayo, concuerda con los tiempos prolongados obtenidos en otras investigaciones, para desechos mineros (7).

Investigaciones recientes revelan el hecho de que la toxicidad del cianuro consiste en inhibir la respiración de los microorganismos. Posiblemente esta es la razón por la que los microorganismos degradadores del cianuro necesitan un tiempo prolongado para su desarrollo (13).

Los microorganismos que biodegradan el cianuro tienen sistemas enzimáticos específicos que les permiten desarrollarse en ambientes con alta concentración de cianuro. Durante la biodegradación, la cianuro hidratasa convierte al cianuro en formamida, que finalmente es convertida a dióxido de carbono $\left(\mathrm{CO}_{2}\right)$ y amoniaco $\left(\mathrm{NH}_{3}\right)$. Otras enzimas como la beta-cianoalamina y la cianuro monoxidasa también pueden biodegradar el cianuro a otras sustancias más simples que no contaminen. Además, algunas bacterias transforman directamente el cianuro a $\mathrm{CO}_{2}$ y $\mathrm{NH}_{4}$ por medio de la cianuro dioxigenasa, sin la formación de intermediaros (I4).

Para el desarrollo, obtención y adaptación del consorcio microbiano, degradador del cianuro, se aprovechó la capacidad de los microorganismos de utilizar los compuestos cianurados como fuente de carbono y nitrógeno y de esta manera se convierten los desechos tóxicos del cianuro en sustancias que ya no contaminan.

La selección de un consorcio de microorganismos más fuerte y eficientes en degradar el cianuro, se logró por el método del cultivo continuo. Por este método los microorganismos dominantes se seleccionaron por su afinidad por el sustrato. En este caso, la concentración del desecho del cianuro (sustrato selectivo), aumentó la velocidad específica de crecimiento del consorcio de microorganismos degradadores de cianuro, con respecto a la velocidad de crecimiento de otros microorganismos que pudieran crecer en el mismo medio (I5).

La liofilización para conservar el consorcio de microorganismos degradadores del cianuro se llevó a cabo en dos etapas. Una primera etapa de congelación lenta, a de 0 a -3 grados centígrados, por 24 horas en donde el agua que contenía el extracto de microorganismos se solidificó. La congelación es de vital importancia para que los solutos y el agua no congelada formen una fase vítrea que permita conservar las características y la estructura del producto final liofilizado. Esta última queda fijada durante esta etapa de congelación ( I6).

La segunda etapa de la liofilización consistió en la sublimación del agua congelada en el extracto denominado secado primario. Para esta etapa se eligieron presiones que van desde 0,0250 a 0,0054 milibares que corresponden a temperaturas durante la sublimación de -35 a -65 grados centígrados.

La etapa del secado primario se realizó por debajo del punto triple del agua, para evitar el paso de esta por la fase líquida, de tal manera que pasara directamente de la fase sólida a la fase de vapor (sublimación). Esto permite conservar la estructura inicial de producto así como sus características, químicas y biológicas ( I6).

Durante el proceso de secado por sublimación se logró eliminar del 95 al 98\% del agua en el extracto de microorganismos.

Después del secado primario (por sublimación) se realizó un segundo secado, denominado secado secundario el cual se llevó a cabo a 20 grados centígrados por lapsos de 8 a 10 horas. Esto se realizó con el propósito de eliminar el agua que quedó fijada o ligada por adsorción durante el proceso de congelación ya que las moléculas del agua libre, es decir no fijada a la estructura de los microorganismos, se eliminaron en el secado primario.

Se utilizó caldo lactosado y gelatina como medios protectores de las bacterias, durante todos los ensayos de liofilización. Cuando se analizó la influencia del medio protector en la liofilización, se pudo apreciar que con el caldo lactosado se obtuvieron buenos resultados de liofilización, pero presentó la desventaja de que el producto final fue difícil de solubilizar durante la recuperación. Esto posiblemente por la descomposición de la lactosa durante el proceso de liofilización, lo que causa que la lactosa forme una capa externa impermeable en el liofilizado que no permite una buena rehidratación del producto (16). 
Sin embargo, al emplear la gelatina como medio protector se obtuvo un producto final que presentó excelentes características de solubilidad, siendo esta prácticamente instantánea.

El uso de protectores es muy importante, por cuanto la liofilización tiene limitaciones durante la etapa de congelación, debido a la formación de cristales de hielo que pueden romper las células y a su vez causar efectos negativos al microorganismo en la etapa de la rehidratación para su reactivación (17).

Tanto la gelatina como el caldo lactosado son medios protectores altamente hidrofilicos. Ambos medios protegen a los microorganismos de los daños producidos por la congelación en la membrana y pared celular. La gelatina es un medio protector no permeable, que se adsorbe en la superficie celular e incrementa la viscosidad local, lo cual mantiene el hielo en forma amorfa y evita el daño mecánico. A diferencia del caldo lactosado que es un medio protector semipermeable que forma, entre la pared y la membrana celular, una capa que protege la célula del daño mecánico ( | 8).

El porcentaje de humedad de los productos finales para la segunda etapa de la liofilización, tanto para el secado primario como el secundario, se resumen en los cuadros 4 y 5 , presentados en el apartado de resultados.

Como se observa en los cuadros, los productos liofilizados indican que la gelatina como medio protector dio mejores resultados en cuanto al porcentaje residual obtenido; esto indica que este medio es muy importante para la conservación prolongada del producto, por varios años. Esta humedad del producto final corresponde a la humedad residual que del todo no puede eliminarse durante el proceso de liofilización ya que de ser eliminada se corre el riesgo de alterar las características químicas y biológicas de los liófilos (19).

Como se mencionó anteriormente, la humedad residual de los liófilos es de vital importancia para su conservación y para mantener la actividad de los microorganismos a largo plazo ya que, entre menor se la humedad, mayor será el tiempo de conservación.

Por otro lado, el contenido de humedad residual es necesario para mantener la actividad de los microorganismos porque las proteínas y péptidos de estos requieren de cierta cantidad de agua para mantener su estructura secundaria y terciaria (20).

La recuperación de los liofilizados se realizó a los seis meses de preservación y almacenamiento a temperatura ambiente. En el cuadro 6 del apartado de resultados se muestran los datos de recuento heterótrofo los cuales representan recuperaciones superiores del $70 \%$ al $80 \%$ y que de acuerdo con lo indicado por M.D. García y F. Uruburú, permite concluir que la liofilización fue exitosa (2I).

Durante la recuperación del liofilizado se logró incrementar la sobrevivencia de los microorganismos rehidratándolos en caldo lactosado y en gelatina; los mismos medios que se usaron para el crecimiento inicial de las células. De esta forma, al mantenerse elevada la presión osmótica durante la rehidratación se logra que la misma se dé en forma lenta. Por otro lado,el crecimiento celular, después de la rehidratación, tiene una fase de retardo extendida, que se puede reducir si se emplea para el crecimiento un medio de igual composición que el que da óptimo desarrollo pero disminuyendo su concentración original entre un 25 y un 50\% (22). Por esta razón la recuperación y rehidratación del consorcio microbiano se realizó en caldo lactosado y gelatina al 10\% en lugar de caldo lactosado y gelatina al 20\%, concentración que se utilizó para el crecimiento inicial del consorcio de microorganismos (22) (23).

Entre los factores más importantes por los que se obtuvieron tan buenos porcentajes de recuperación se pueden mencionar los siguientes (2I):

- El empleo de protectores como la gelatina y el caldo lactosado protegieron adecuadamente a los liófilos de las condiciones drásticas de congelación y presión.

- Las presiones durante el proceso de sublimación del hielo fueron entre 0,250 a 0,0054 milibares, estas condiciones de presión hacen que el proceso de sublimación sea lento con lo que se obtienen liófilos con mejores características de recuperación.

- La alta densidad celular del extracto del consorcio microbiano que se sometió a liofilización que fue superior a $10^{10}-10^{11}$ células/ $100 \mathrm{ml}$, (ver cuadro 5). A altas concentraciones se obtienen mejores productos liofilizados con una buena capacidad de recuperación y de actividad. 
- El grado de secado secundario alcanzado el cual fue inferior al 2\%. Esta agua residual protege de daños a la pared de las células microbianas.

- La rehidratación lenta al diminuir la concentración del medio de rehidratación en un $50 \%$ y no rehidratando directamente en agua destilada.

Los resultados de la cuantificación del cianuro, antes y después del tratamiento total de 15 días, se muestran en el cuadro 7. Puede observarse que las remociones de cianuro fueron en todos los casos superiores al 95\%. Estas altas remociones del cianuro se deben al proceso de adaptación del consorcio que se llevó a cabo hasta la tercera generación con lo que se logró microorganismos más fuertes y más eficientes en la remoción del cianuro (22).

El consorcio de microorganismos demostró tener alta eficiencia en degradar cianuro, en concentraciones superiores a las reportadas por Restrepo et al. en estudios similares. (7).

\section{Conclusiones}

- La adaptación del consorcio microbiano hasta la tercera generación, permitió obtener microorganismos más fuertes y más eficientes en la remoción del cianuro. Se logró alcanzar eficiencias de remoción del 95 al 98\%.

- El consorcio microbiano autóctono obtenido para degradar cianuro demuestra eliminar el cianuro de manera eficiente y económica, con tiempos de retención cortos de 15 días.

- La alta densidad celular del extracto del consorcio microbiano superior a $10^{10}-10^{11}$ células/l00 ml y el proceso de sublimación lento a presiones entre 0,250 a 0,0054 milibares fueron las razones para que se obtuvieran liófilos con excelentes características de recuperación entre $70 \%$ y $80 \%$.

- Las concentraciones de cianuro eliminadas por el consorcio microbiano (2500 mg/L), son superiores a las reportadas por estudios similares (7).

- La lixiviación previa del desecho de cianuro es indispensable para el tratamiento aplicando el consorcio microbiano.

\section{Recomendaciones}

Entre las principales recomendaciones, para proyectos futuros, pueden resumirse las siguientes:

- Realizar estudios de recuperación de los liofilizados de microorganismos del cianuro para determinar su viabilidad a los 2, 4, 8, 12, 16 y 20 años.

- Seguir en contacto con la Compañía Infinito para ofrecer y comercializar el consorcio biodegradador del cianuro.

- Impartir un taller de liofilización por año para motivar a los estudiantes de Ingeniería Ambiental y Biotecnología del TEC para que realicen trabajos de graduación en el campo de los desechos tóxicos y otros.

- Seguir la metodología de obtención de microorganismos afines a un sustrato para obtener microorganismos degradadores de desechos como: desechos lácteos, grasa láctea, desechos del petróleo, lodos de tanques sépticos y lagunas en general.

\section{Bibliografía}

(I) Pavas, E \& Giraldo, C. (2005). Proceso Acoplado físicoquímico y biotecnológico para el tratamiento de aguas residuales procesos Ambientales y Biotecnológicos. Universidad Colombia, ISSN 1692-0694. Noviembre del 2005. Documento 38-I 12005.

(2) LaGrega, M; et al. (1996). Gestión de Residuos Tóxicos. Tratamiento, eliminación y recuperación de suelos. Volumen II. Mc Graw Hill. España.

(3) Castro, M. L \& Gonzáles, F. D. (2002). Eficiencia de la Biodegradación de Cianuro por un consorcio microbiano aislado de aguas superficiales de quebradas que alimentan el río Llaucano. Universidad Pedro Ruiz Gallo. Perú.

(4) American Water Works Association, American Public Health Association, Water Polution Control Faderation. (1992). Métodos Normalizados para el Análisis de Aguas potables y Residuales. 19th edición. Ediciones Díaz De Santos. España.

(5) Voget, C. (2005). Conservación de cultivos para la biotecnología y la industria, cátedras. Química.unlp.edu. ar. Curso CABBIO, 2005. Consultado el 15 de junio del 2010. Obtenido desde: http://catedras.quimica. unlp.edu.ar/ingenieriabioquimicalyll/metconsmicro.ppt. 
(6) Zamora, L. M. (2003). Aislamiento, identificación y conservación de cultivos lácticos. Tesis Doctoral, Universidad de Girona. España.

(7) Restrepo, et al. (2006). Degradación Microbiana de Cianuro procedente de plantas de Beneficio de oro, mediante una cepa nativa de P. fluorecens. Universidad Nacional de Colombia. Instituto de Minerales, Universidad de Antioquia. Maestría en Ingeniería Ambiental. Colombia.

(8) Eckenfelder,W. (2000). Industrial water pollution control. USA. Mc Graw Hill.

(9) Logsdon, M. J; Kagelstein, K. \& Mudder, T. I. (200I). El Manejo del Cianuro en la Extracción de Oro. Consejo Nacional de Metales y Medio Ambiente. USA.

Consultado el: 24 de marzo del 2009. Obtenido desde: http://www.aage.org.ar/manejodelcianuro.pdf.

(I0) Blanco, D \& Rendueles, M. (200I). Reducción del impacto ambiental en el desarrollo de nuevos proyectos de minería de oro. Áreas Técnicas.

Consultado el 25 de marzo del 2009.

Obtenido desde: http://www.unizar.es/aeipro/finder/ MEDIO\%20AMBIENTE

(I I) Guerrero, J.J. (2003). Cianuro: Degradación Biológica. Perú. Consultado el 25 de marzo del 2009. Obtenido desde:http://www.unt.edu/resource/02cyanidefeature. htm.

( I2) Logsdon, M. J. El manejo del cianuro en la extracción de oro. Consultado el 22 de mayo del 2009.

Obtenido desde: http://www.aage.org.ar/ manejodelcianuro.pdf.

( 13 ) Guerrero,J. Cianuro:Toxicidad y Destrucción Biológica. Revista El ingeniero de Minas. Año X, n 35. Perú 2003. Consultado el 28 de octubre del 2009. Obtenido desde: //www.unt.edu/resource/02cyanidefeature.htm.

(14) Guerrero, J. (2004). Biodegradación del Cianuro. Congreso Nacional de Ciencias Ambientales. Universidad Nacional Federico Villareal, Perú.

(15) Microbiología Industrial. (1981). Selección, mantenimiento y mejoramiento de microorganismos de interés industrial. Capítulo 3. Biotechnology. Vol. I. Microbial Fundamentals. Ed. H.J. Rehm, G. Reed, Verlag Chemie.

(16) Zamora, L. M. (2003). Aislamiento, identificación y conservación de cultivos lácticos. Tesis Doctoral, Universidad de Girona. España.
(17) García, M. D. \& Uruburu, F. Colección Española de Cultivos Tipo (CECT). Universidad de Valencia. 46100 Burjassot (Valencia). Consultado el 9 de febrero del 2010 . Obtenido desde: http://catedras.quimica.unlp. edu.ar/ingenieriabioquimicalyll/seminario3.doc

(18) Voget, C. (2005). Conservación de cultivos para la biotecnología y la industria. catedras.quimica.unlp. edu.ar. Curso CABBIO. Obtenido desde: http:// catedras.quimica.unlp.edu.ar/ingenieriabioquimicalyll/ metconsmicro.ppt

(19) Freire, J.R. \& Sato, M.L. (1999). Conservación de Cultivos de Rizobios. Revista Latinoamericana de Biología. Vol.4l:34-4l.

(20) Fernández, A. General Information to Pharma 2. Módulo V, Liofilización: Un poco de historia. Consultado el 13 de junio del 2010 . Obtenido desde: http://personal.us.es/ mfarevalo/recursos/tec_far/liofilizacion.pdf

(2I) García, M. D. \& Uruburu, F. Colección Española de Cultivos Tipo (CECT). Universidad de Valencia. 46100 Burjassot (Valencia). Consultado el 9 de febrero del 2010. Obtenido desde: http://catedras.quimica.unlp. edu.ar/ingenieriabioquimicalyll/seminario3.doc

(22) Microbiología Industrial. (1981). Selección, mantenimiento y mejoramiento de microorganismos de interés industrial. Capítulo 3. Biotechnology. Vol. I. Microbial Fundamentals. Ed. H.J. Rehm, G. Reed, Verlag Chemie.

(23) S. Borrego et al. (200I). Comportamiento de Tres Mutantes de micobacterias productoras de precursores esteroides conservadas por liofilización. Biotechnology Microbial Fundamentals. Ed. H.J. Rehm, G. 200I, Brasil.

(24) Sepúlveda, V \& Velasco, T. Tecnologías de remediación para suelos contaminados. México: Instituto Nacional de Ecología, 2002. Consultado 28 de mayo del 2010. Obtenido desde: http://www2.ine.gob.mx/ publicaciones/libros/372/tecnomexico.html

(26) Tchobanoglous, G \& Burton, F. (1995). Ingeniería de Aguas Residuales: Tratamiento, Vertido y Reutilización. Tercera Edición. Madrid McGraw Hill Interamericana de España S.A, España. 1012 pp.

(28) División de Protección al Ambiente Humano. Reglamento de Vertido y Reuso de Aguas Residuales, decreto, No 3360 I-MINAE-MINSALUD, Ministerio de Salud, Costa Rica, 2007. 